\title{
Is There an Easy, Effective, Efficient, and Inexpensive Technique to Reduce Pain of Arterial Punctures?
}

This issue of Respiratory CARE features a well-written paper by Haynes ${ }^{1}$ on the use of cryoanalgesia, specifically by application of an ice bag, to reduce pain associated with arterial puncture. Arterial puncture for blood gas (ABG) analysis is a common procedure in a variety of clinical settings, and although it is often performed in sedated patients on mechanical ventilation, it is also frequently performed on patients who are awake, and it can cause significant pain and discomfort. ${ }^{2}$ Perhaps due to the often time-sensitive nature of $\mathrm{ABG}$ analysis, the use of effective analgesic techniques for this procedure are not routinely employed in many settings. ${ }^{3}$ However, the lack of adequate analgesia can lead to unnecessary anxiety and morbidity and may also complicate successful completion of the procedure. Therefore, further study of effective and efficient means of providing adequate pain reduction for arterial puncture is clearly warranted.

This was a randomized controlled trial of adult outpatients presenting to a pulmonary function laboratory for ABG collection. All blood draws were from the radial artery. The treatment group had ice bags applied to their wrists for $3 \mathrm{~min}$ before the puncture, whereas the control group had no ice bags. Pain was measured by a $100-\mathrm{mm}$ visual analog scale. Haynes found a reduction in reported pain in the treatment group (mean visual analog scale score of $13.8 \pm 16.9$ vs $25 \pm 23 \mathrm{~mm}$ ). Subjects were also stratified as either naive (unable to recall a previous arterial puncture procedure) or experienced, and the analysis showed a significant reduction in reported pain in the naive group (mean visual analog scale score of $11 \pm 14.3 \mathrm{vs}$ $26.5 \pm 25 \mathrm{~mm}$ ) and a trend toward reduction in reported pain in the experienced group (mean visual analog scale score of $15.9 \pm 18.9$ vs $25.1 \pm 22 \mathrm{~mm}$ ). As noted by the author, the lack of statistical significance in the experienced group likely represents inadequate power of the study to detect a clinically important difference in the sub-

\footnotetext{
The authors have disclosed no conflicts of interest.

Correspondence: S David McSwain MD MPH, Department of Pediatrics, Division of Pediatric Critical Care Medicine, Medical University of South Carolina, 135 Rutledge Avenue, MSC 566, Charleston, SC 29425. Email: mcswains@musc.edu.
}

DOI: $10.4187 /$ respcare. 03865 groups. A power analysis was conducted before the study, but only for the total group analysis. However, apprehension regarding the procedure or memory of previous pain associated with an arterial puncture may have also affected the scores of the experienced group. Haynes also found no difference in procedure success rate between groups: $85 \%$ of the treatment group successfully completed the procedure on the first attempt versus $82.5 \%$ of the control group. In addition, only 3 of 42 subjects in the treatment group could not tolerate the 3-min ice application.

Although this topic has not been extensively studied, published studies provide useful comparisons. France et $\mathrm{al}^{4}$ conducted a randomized controlled trial of local anesthesia for arterial puncture with subcutaneous lidocaine, with ethyl chloride cooling spray (which has demonstrated efficacy for reduction of pain in venipuncture and peripheral venous cannulation ${ }^{5,6}$ ), or without anesthesia on 54 adult

See the Original Study on Page 1

subjects in 2 urban emergency departments. A 100-mm visual analog scale was used to compare pain experienced in the 3 study groups. The authors found that subcutaneous lidocaine, injected $2 \mathrm{~min}$ before arterial puncture, was effective in reducing pain (mean visual analog scale score of $10.2 \mathrm{~mm}, 95 \%$ CI $4.8-16.3$ ) compared with no anesthesia (mean visual analog scale score of $23.4 \mathrm{~mm}, 95 \%$ CI $11.7-$ 35.0), but that ethyl chloride cooling spray did not impact pain experienced (mean visual analog scale score of $23.9 \mathrm{~mm}, 95 \%$ CI 12.4-35.5). However, they also found that pain associated with lidocaine administration was similar to that of the arterial puncture itself (mean visual analog scale score of $22.0 \mathrm{~mm}, 95 \%$ CI 10.9-33.7). The authors concluded that the benefit of local lidocaine injection was limited due to injection pain.

Other studies have evaluated the use of topical anesthetics to reduce the pain of arterial punctures and have shown effectiveness. Aaron et $\mathrm{al}^{7}$ conducted a randomized double-blind placebo-controlled trial of topical tetracaine before arterial puncture. The gel was applied to the injection site $45 \mathrm{~min}$ before puncture in subjects referred for elective ABG draws. The primary outcome was the subjects' pain perception as measured by the $100-\mathrm{mm}$ visual 
analog scale. Of the 50 subjects randomized in the trial, researchers found that the group receiving tetracaine reported a mean pain score of $26.2 \pm 32.6 \mathrm{~mm}$, whereas the placebo group reported a mean score of $23.8 \pm 27.4 \mathrm{~mm}$. The results of this study suggested that the use of tetracaine gel before $\mathrm{ABG}$ was ineffective in decreasing perceived procedure-associated pain. In addition, the study concluded that the use of tetracaine did not significantly improve procedural success by way of vasodilatory response, as was hypothesized by the authors. Twenty-five percent of the tetracaine-treated group required more than one puncture to obtain the arterial blood sample versus $15 \%$ of the placebo cohort. In addition, the 45-min application time for adequate effect of topical tetracaine may not only be prohibitively long in the majority of settings in which ABG sampling is needed, but may have contributed to increased pre-procedure anxiety in this study. The authors concluded that tetracaine gel neither improved analgesia nor facilitated ease of arterial puncture.

More recently, the concept of jet injection for analgesic delivery has gained popularity. Jet injectors are a relatively new needle-free technology. The design uses jet injectors driven by carbon dioxide or springs that force, under pressure, the delivery of medication through the skin in a fraction of a second with almost no perception of pain. Moreover, these delivery devices are also capable of dispersing medications more broadly than the traditional needle/syringe injections, thus making drug absorption more rapid. ${ }^{8}$ A randomized controlled trial conducted by Hajiseyedjavady et $\mathrm{al}^{9}$ examined the use of jet injection of $2 \%$ lidocaine as a means of pain reduction before ABG. Forty-two subjects were randomized into 2 groups, with group $\mathrm{A}$ receiving the $2 \%$ lidocaine by jet injection and group $\mathrm{B}$ receiving $1 \mathrm{~mL}$ of $2 \%$ lidocaine gel by topical application $2 \mathrm{~min}$ before ABG sampling. Pain was assessed using a $10-\mathrm{cm}$ visual analog scale, with a rating of $0 \mathrm{~mm}$ being the absence of pain. Results yielded a mean visual analog scale score of $1.29 \pm 0.90 \mathrm{~mm}$ for the group receiving lidocaine by jet injector versus a mean visual analog scale score of $4.19 \pm 1.43 \mathrm{~mm}$ for the control group $(P<.001)$. Additionally, the number of attempts in group A was significantly lower than that in group B $(1.29 \pm 0.46$ vs $2.1 \pm 0.12, P=.009)$, and all who performed the procedure reported ease of use with the jet injector. The authors concluded that use of the lidocaine jet injector significantly reduced the perceived pain of those undergoing arterial puncture. However, although the jet injection technique is promising for reducing pain from injections, it is not widely available at this time and would require the purchase and maintenance of specialized devices.

Ice application offers a safe, easy, noninvasive, and inexpensive means of reducing pain associated with arterial puncture, particularly in situations in which intradermal anesthetics are unavailable or the proceduralist is uncomfortable with or unable to administer intradermal injections. It is important to note the exclusion criteria of Haynes' study, particularly the exclusion of patients with Raynaud's disease or scleroderma/CREST (calcinosis, Raynaud's phenomenon, esophageal dysmotility, sclerodactyly, and telangiectasia) syndrome, because ice application in those settings could have significant adverse effects. In addition, ice application in this study was carefully timed with a stopwatch, and subjects were allowed to remove the ice early for significant discomfort, which is important to avoid prolonged or excessive ice exposure leading to tissue injury.

All punctures in this study were performed by the investigator to eliminate technologist technique and skill as a variable. For comparison, the study by France et $\mathrm{al}^{4}$ included arterial punctures performed by 29 different physicians and documented the mean number of attempts for each study group (1.3, 1.2, and 1.1 for the no-anesthetic, lidocaine, and ethyl chloride groups, respectively). Given the similar visual analog scale scores documented with no local anesthesia in the 2 studies, it appears that the proceduralists' skill was not a significant confounder in the France study.

Although France et $\mathrm{al}^{4}$ scored pain associated with the injection of subcutaneous lidocaine and application of ethyl chloride cooling spray, they did not score pain or discomfort associated with application of the ice itself. The visual analog scale score was low for ethyl chloride application (mean visual analog scale score of $12.9 \mathrm{~mm}, 95 \%$ CI 5.5$20.3 \mathrm{~mm}$ ), but the cooling spray did not result in a reduction in pain from the procedure. However, the cooling spray was applied only briefly, until the skin became frosted in appearance, which likely resulted in more superficial cooling than the ice applied in the study by Haynes. ${ }^{1}$ Haynes states that the ice application was tolerated by all but 3 subjects, but a score for their discomfort would help better quantify the benefit of ice application.

This study provides evidence that cryoanalgesia is a safe, inexpensive, easy-to-apply, and readily available method of pre-procedure analgesia with a reasonably rapid onset of action. As long as appropriate procedural guidelines are put in place with regard to exclusion for certain comorbid conditions, patient tolerance of ice application, and avoidance of prolonged ice exposure, the potential benefits to patient comfort appear to outweigh the minimal risks of cryoanalgesia. Given the short duration required for adequate effect, the additional time required for application of this technique should not be prohibitive except in the most severely time-critical situations. As such, this study provides evidence that ice pack cryoanalgesia would be an appropriate addition to routine arterial puncture procedures in a variety of clinical settings. 


\section{Reducing Pain of Arterial Punctures}

S David McSwain MD MPH

Department of Pediatrics Division of Pediatric Critical Care Medicine

Center for Telehealth Medical University of South Carolina Charleston, South Carolina

Brooke E Yeager MSc RRT Center for Telehealth Medical University of South Carolina

Charleston, South Carolina

\section{REFERENCES}

1. Haynes JM. Randomized controlled trial of cryoanalgesia (ice bag) to reduce pain associated with arterial puncture. Respir Care 2015; 60(1):1-5.

2. Turner JS, Briggs SJ, Springhorn HE, Potgieter PD. Patients' recollection of intensive care unit experience. Crit Care Med 1990;18(9): 966-968.
3. Lightowler JV, Elliott MW. Local anaesthetic infiltration prior to arterial puncture for blood gas analysis: a survey of current practice and a randomised double blind placebo controlled trial. J R Coll Physicians Lond 1997;31(6):645-646.

4. France JE, Beech FJ, Jakeman N, Benger JR. Anaesthesia for arterial puncture in the emergency department: a randomized trial of subcutaneous lidocaine, ethyl chloride or nothing. Eur J Emerg Med 2008; 15(4):218-220

5. Armstrong P, Young C, McKeown D. Ethyl chloride and venepuncture pain: a comparison with intradermal lidocaine. Can J Anaesth 1990;37(6):656-658.

6. Selby IR, Bowles BJ. Analgesia for venous cannulation: a comparison of EMLA (5 min application), lignocaine, ethyl chloride, and nothing. J R Soc Med 1995;88(5):264-267.

7. Aaron SD, Vandemheen KL, Naftel SA, Lewis MJ, Rodger MA. Topical tetracaine prior to arterial puncture: a randomized, placebocontrolled clinical trial. Respir Med 2003;97(11):1195-1199.

8. Kale TR, Momin M. Needle free injection technology-an overview. Innov Pharm 2014;5(1):1-8.

9. Hajiseyedjavady H, Saeedi M, Eslami V, Shahsavarinia K, Farahmand $\mathrm{S}$. Less painful arterial blood gas sampling using jet injection of $2 \%$ lidocaine: a randomized controlled clinical trial. Am J Emerg Med 2012;30(7):1100-1104. 